\title{
Role of the Self-Administered, Self-Reported History Questionnaire to Identify Types of Lumbar Spinal Stenosis: A Sensitivity Analysis
}

\author{
Hossein Nayeb Aghaei, Parisa Azimi, Sohrab Shahzadi, Shirzad Azhari, Hassan Reza Mohammadi
}

Department of Neurosurgery, Shahid Beheshti University of Medical Sciences, Tehran, Iran

\section{Study Design: Case-control design.}

Purpose: To evaluate the role of the self-administered, self-reported history questionnaire (SSHQ) in identifying types of lumbar spinal stenosis (LSS).

Overview of Literature: Diagnosis of types of LSS is controversial.

Methods: A total of 235 patients with LSS were asked to respond to the SSHO. All of these patients recovered following surgical treatment. The classification of LSS patients was based on history, physical examinations, and imaging studies. It is considered to be the gold standard. Radicular and neurogenic claudication types of LSS were based on the SSHO developed by Konno et al. Two categories of LSS were determined based on the SSHO tool and gold standard. Finally, a sensitivity analysis was carried out to evaluate the diagnostic value of the SSHO.

Results: The mean age of patients was 59.4 years. According to the criteria for gold standard, patients were diagnosed with the radicular type (n=103), and neurogenic claudication type ( $n=132)$. The questionnaire had desirable sensitivity, specificity, and accuracy in categorizing the two types of LSS: $97.8 \%, 66.6 \%$, and $96.8 \%$ for the radicular type, and $97.0 \%, 80.0 \%$, and $95.7 \%$ for the neurogenic claudication type.

Conclusions: Our findings indicate that the $\mathrm{SSHQ}$ is a reliable and a valid measure and it may be a clinical diagnosis support tool for identifying patients with two types of LSS.

Keywords: Lumbar spinal stenosis; Self-reported history questionnaire; Neurogenic claudication; Radicular pain

\section{Introduction}

Lumbar spinal stenosis (LSS) is a common spine disorder in the elderly, and it includes patients with symptoms related to narrowing of the spinal canal with subsequent neural compression [1]. To establish a diagnosis of LSS, clinical history, physical examination findings, electrodiagnostic test, questionnaires, and radiological changes may be used, either separately or in combination [2], However, there are still no widely accepted diagnostic or classification criteria for the diagnosis of LSS [3]. Recently, for the diagnosis and categorization of LSS patients, the self-administered, self-reported history questionnaire (SSHQ) has been developed. The SSHQ is a simple, reliable, and a user-friendly clinical tool for diagnosis of LSS in patients, as suggested previously [4]. The aim of this

Received Jan 19, 2015; Revised Feb 4, 2015; Accepted Feb 11, 2015

Corresponding author: Parisa Azimi

Functional Neurosurgery Research Center of Shohada Tajrish Hospital,

Shahid Beheshti University of Medical Sciences, Tehran, Iran

Tel: +98-21-2274-9204, Fax: +98-21-8826-5188, E-mail: parisa.azimi@gmail.com 
study was to evaluate the role of the SSHQ in identifying patients with two types of LSS such as the radicular and neurogenic claudication types.

\section{Materials and Methods}

\section{Patients and data collection}

Between June 2009 and January 2013, a sample of LSS patients who attended the neurosurgery clinic of a teaching hospital in Tehran, Iran, was studied. The LSS patients were diagnosed and categorized into the radicular and neurogenic claudication types based on history, clinical symptoms, neurological examinations, and imaging studies including plain radiography, magnetic resonance imaging (MRI) and computer tomography (CT) of the lumbar spine before surgery. All of the patients had typical symptoms of LSS, such as leg pain and/or numbness and neurogenic claudication. The diagnosis was established by more than one spine surgeon in all of the cases, and their opinions were considered as the gold standard for diagnosis of LSS. In the absence of a universally accepted gold standard in diagnosis of LSS, the impression of expert physicians provides a practical method of establishing a clinical diagnosis. The stenosis level(s) were studied on the CT or MRI images. All of the patients recovered following surgical treatment. There were no limitations on patient selection with respect to mild, moderate, or severe LSS or age. The exclusion criteria were diabetic neuropathy, peripheral vascular disease, cervical myelopathy, previous surgery, inflammatory disorders, and other types of low back pain such as lumbar disc herniation.

Data including gender, age, and body weight were recorded. The duration of symptoms (month) and walking distance (meter) were assessed.

\section{Additional measure (SSHQ)}

The SSHQ is a specific diagnostic tool for identifying patients with LSS and then distinguishing between patients with radicular type and those with neurogenic claudication. It consists of ten questions. Each item is scored using a yes/no response. Answering "yes" to questions 1 through 4 and "no" to questions 5 through 10 indicates

Patient name:

Date:

This list contains some sentences that people have used to describe themselves when they have lumbar spinal stenosis. As you read the list, think of yourself. When you read a sentence that describes you, please circle "yes." If the sentence does not describe you, please circle "no."

\begin{tabular}{|c|c|c|}
\hline 1. Numbness and/or pain in the thighs down to the calves and shins. & Yes & No \\
\hline $\begin{array}{l}\text { 2. Numbness and/or pain increase in intensity after walking for a while, but are relieved by } \\
\text { taking a rest. }\end{array}$ & Yes & No \\
\hline $\begin{array}{l}\text { 3. Standing for a while brings on numbness and/or pain in the thighs down to the calves and } \\
\text { shins. }\end{array}$ & Yes & No \\
\hline 4. Numbness and/or pain are reduced by bending forward. & Yes & No \\
\hline 5. Numbness is present in both legs. & Yes & No \\
\hline 6. Numbness is present in the soles of both feet. & Yes & No \\
\hline 7. Numbness arises around the buttocks. & Yes & No \\
\hline 8. Numbness is present, but pain is absent. & Yes & No \\
\hline 9. A burning sensation arises around the buttocks. & Yes & No \\
\hline 10. Walking nearly causes urination. & Yes & No \\
\hline
\end{tabular}

Fig. 1. The English original version of the self-administered, self-reported history questionnaire. Based on the authors (6) a score of 4 points on questions 1 through 4 indicates the presence of lumbar spinal stenosis (LSS); a score of 4 on questions 1 through 4 and "no" to questions 5 through 10 indicates the radicular type of LSS. Answering "yes" to at least one of questions 1 through 4, and at least two of questions 5 through 10 indicates the neurogenic claudication (referred as cauda equina in their manuscript) type of LSS. 
the radicular type of LSS. Answering "yes" to at least one of the questions 1 through 4 , and at least two of the questions 5 through 10 indicates the neurogenic claudication (referred to as cauda equina in their manuscript) type of LSS (Fig. 1) [4]. The patients completed the SSHQ before surgery.

\section{Statistical analysis}

Sensitivity analysis: First, patients were classified based on their SSHQ classification as proposed by Konno et al. [4]. Then, the actual classification was based on history, physical examinations, and MRI as the gold standard for classification of patients into the radicular and neurogenic claudication types.

Finally, the results obtained from the estimated and actual categorizations were compared using the sensitivity analysis. In fact, with respect to the actual classification score for each case, the estimated classifications were tested and designated as true positive, true negative, false positive, or false negative in order to calculate the sensitivity, specificity, and accuracy for the estimated classifications [5].

All statistical analyses were performed using the PASW ver. 18.0 (SPSS Inc., Chicago, IL, USA).

\section{Ethics}

The Ethics Committee of Shahid Beheshti University of Medical Sciences, Tehran, Iran, approved the study.

\section{Results}

The characteristics of the LSS patients and their types of disease based on the SSHQ are shown in Table 1. The sensitivity and specificity, as well as the positive and negative predictive values regarding the diagnostic success of categorization of different types of LSS based on the SSHQ, were calculated. The sensitivity, specificity, and accuracy of the SSHQ in diagnosing the radicular type $(97.8 \%$, $66.6 \%$, and $96.8 \%$ ) and the neurogenic claudication type (97.0\%, 80.0\%, and 95.7\%) of LSS were found to be desirable. The positive and negative predictive values of the SSHQ were $98.9 \%$ and $50.0 \%$ for the radicular type and $99.2 \%$ and $44.5 \%$ for the neurogenic claudication type groups. The results for each group are shown in separate tables (Tables 2, 3).
Table 1. The characteristics of the study sample $(n=235)$

\begin{tabular}{|c|c|}
\hline Characteristic & Value \\
\hline Age group (yr) & $59.4 \pm 11.3(32-84)$ \\
\hline \multicolumn{2}{|l|}{ Sex } \\
\hline Male & $98(41.7)$ \\
\hline Female & $137(58.3)$ \\
\hline Body weight (kg) & $3.3 \pm 10.6$ \\
\hline Walking distance (m) & $362.1 \pm 256$ \\
\hline \multicolumn{2}{|l|}{ Lumbar stenosis level (\%) } \\
\hline One & $25(10.6)$ \\
\hline Two & $89(37.9)$ \\
\hline Three & $78(33.2)$ \\
\hline Four & $43(18.3)$ \\
\hline \multicolumn{2}{|c|}{ Types of $\mathrm{LSS}_{\text {as }}$ classified by $\mathrm{SSH}^{\mathrm{a}}$} \\
\hline Radicular type & $95(40.4)$ \\
\hline Neurogenic claudication type & $140(59.6)$ \\
\hline \multicolumn{2}{|c|}{ Types of LSS as classified by gold standard ${ }^{\text {b) }}$} \\
\hline Radicular type & $103(43.8)$ \\
\hline Neurogenic claudication type & $132(56.2)$ \\
\hline \multicolumn{2}{|c|}{ 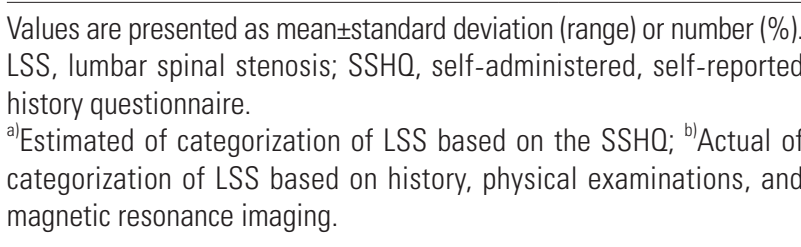 } \\
\hline
\end{tabular}

\section{Discussion}

The results obtained in the present study showed that the SSHQ tool is reliable and valid, and it may improve the accuracy in categorizing different types of LSS.

Konno et al. [4] developed a simple clinical diagnostic support tool to identify patients with LSS [6]. The SSHQ was developed successfully as a diagnostic support tool to identify patients with LSS and to differentiate between patients with radicular compression and neurogenic claudication (referred to as "cauda equina" symptoms in their manuscript). They showed that this tool satisfactorily assessed the diagnostic value of the history of the patient for categorization of LSS. They found that the sensitivity and specificity of the SSHQ were $84 \%$ and $78 \%$, respectively, in the validation data set, which is in line with our findings. In the current study, a sensitivity analysis was performed, and it showed that the SSHQ is a sensitive questionnaire for discriminating between patients. We could not find 
Table 2. Two-by-two matrices of the relationship between the estimated radicular type and the actual radicular type (sensitivity analysis)

\begin{tabular}{lccc} 
& & & Actual radicular type $^{\text {b) }}$ \\
\cline { 2 - 4 } Estimated radicular type & & Negative & Total \\
Positive & Positive & 1 (false positive) & 91 \\
Negative & 90 (true positive) & 2 (true negative) & 4 \\
Total & 2 (false negative) & 3 & 95 \\
\hline
\end{tabular}

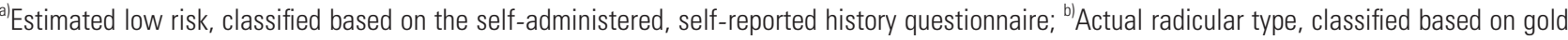
standard. Sensitivity=90/(90+2)=97.8\%; Specificity=2/(2+1)=66.6\%; Accuracy=(90+2)/(90+1+2+2)=96.8\%; Positive predictive value=(true positive) $/$ (true positive+false positive)=90/91=98.9\%; Negative predictive value=(true negative)/(true negative+false negative) $=2 / 4=50 \%$.

Table 3. Two-by-two matrices of the relationship between the estimated cauda equina type and the actual neurogenic claudication type (sensitivity analysis)

\begin{tabular}{|c|c|c|c|}
\hline \multirow{2}{*}{ Estimated neurogenic claudication type ${ }^{\text {a) }}$} & \multicolumn{3}{|c|}{ Actual neurogenic claudication type ${ }^{\text {b) }}$} \\
\hline & Positive & Negative & Total \\
\hline Positive & 130 (true positive) & 1 (false positive) & 131 \\
\hline Negative & 5 (false negative) & 4 (true negative) & 9 \\
\hline Total & 135 & 5 & 140 \\
\hline
\end{tabular}

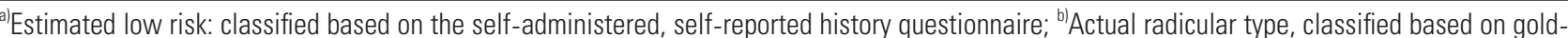
standard. Sensitivity=130/(130+4)=97.0\%; Specificity $=4 /(4+1)=80.0 \%$; Accuracy= $(130+4) /(130+1+4+5)=95.7 \%$; Positive predictive value=(true positive)/(true positive+false positive) $=130 / 131=99.2 \%$; Negative predictive value=(true negative)/(true negative+false negative)=4/9=44.5\% .

any other studies on sensitivity analysis of the SSHQ score in order to compare with our results. However, these results suggest that the SSHQ can be useful for identifying the types of LSS.

There are no established standardized diagnostic criteria for comprehensive evaluation based on the radiographic findings and clinical indications [7]. Various diagnostic support tools for LSS such as the SSHQ have been developed. Sugioka et al. [8] prospectively assessed 468 patients with lower extremity symptoms to develop and validate a prediction rule for the identification of LSS based on self-reported patient information alone. They concluded that this self-administered questionnaire could be useful for improving the accuracy of diagnosis of spinal stenosis, and in particular to rule out LSS. Konno et al. $[4,6]$ reported results from two studies suggesting that a constellation of variables could contribute to the diagnosis of LSS. After evaluating 468 patients, using univariate and multiple regression analyses, several key determinants were found to be predictive of LSS. The SSHQ was developed to improve the accuracy of the diagnosis of LSS, and also to differentiate between the two types of
LSS, radicular type and cauda equina type [4]. However, there is insufficient evidence to make a recommendation for or against the use of self-administered questionnaires such as the SSHQ to improve accuracy of the diagnosis of spinal stenosis [9]. In this study, we observed that the SSHQ is a sensitive measure for categorization of LSS patients. Therefore, misdiagnosis of LSS at the primary care levels can be minimized and patients have a greater chance of gaining health care access; thus, improving the accuracy of the diagnosis and leading to improved quality of patient care $[4,10]$. The use of this simple measure is recommended in order to increase the diagnostic success of categorization of types of LSS especially in teaching hospitals and it might be more useful for the clinicians or patients.

This study has several limitations. There is no gold standard in the diagnosis of LSS; however, we believe that expert physicians provide a practical decision-making solution. We did not identify all possibly relevant variables that need to be assessed for making the diagnosis in these patients. Future studies are needed to clarify this issue. Finally, we carried out a number of limited tests to per- 
form this validation study. In future, it might be necessary to perform other tests to establish stronger psychometric indexes for the SSHQ in primary care settings.

\section{Conclusions}

Our findings indicate that the SSHQ is a reliable and a sensitive measure and it may be a clinical diagnosis support tool for categorizing patients with different types of LSS.

\section{Conflict of Interest}

No potential conflict of interest relevant to this article was reported.

\section{Acknowledgments}

The authors thank the staff of the Neurosurgery Unit at Imam-Hossain Hospital, Tehran, Iran.

\section{References}

1. Gevirtz C. Update on treatment of lumbar spinal stenosis. Part 1: defining the problem, diagnosis, and appropriate imaging. Top Pain Manag 2010;25:1-5.

2. Genevay S, Atlas SJ. Lumbar spinal stenosis. Best Pract Res Clin Rheumatol 2010;24:253-65.

3. Genevay S, Atlas SJ, Katz JN. Variation in eligibility criteria from studies of radiculopathy due to a herniated disc and of neurogenic claudication due to lumbar spinal stenosis: a structured literature review. Spine (Phila Pa 1976) 2010;35:803-11.

4. Konno S, Kikuchi S, Tanaka Y, et al. A diagnostic support tool for lumbar spinal stenosis: a self-administered, self-reported history questionnaire. BMC Musculoskelet Disord 2007;8:102.

5. Kirkwood BR, Sterne JA. Essential medical statistics. 2nd ed. Oxford: Blcakwell Science Ltd; 2003.

6. Konno S, Hayashino Y, Fukuhara S, et al. Development of a clinical diagnosis support tool to identify patients with lumbar spinal stenosis. Eur Spine J 2007;16:1951-7.

7. Yabuki S, Fukumori N, Takegami M, et al. Prevalence of lumbar spinal stenosis, using the diagnostic support tool, and correlated factors in Japan: a population-based study. J Orthop Sci 2013;18:893-900.

8. Sugioka T, Hayashino Y, Konno S, Kikuchi S, Fukuhara S. Predictive value of self-reported patient information for the identification of lumbar spinal stenosis. Fam Pract 2008;25:237-44.

9. North American Spine Society. Evidence-based clinical guidelines for multidisciplinary spine care: diagnosis and treatment of degenerative lumbar spinal stenosis [Internet]. Burr Ridge, IL: North American Spine Society; c2011 [cited 2015 May 20]. Available from: https://www.spine.org/Documents/ResearchClinicalCare/Guidelines/LumbarStenosis.pdf.

10. Ohtori S, Sekiguchi M, Yonemoto K, Kakuma T, Takahashi K, Konno S. Awareness and use of diagnostic support tools for lumbar spinal stenosis in Japan. J Orthop Sci 2014;19:412-7. 